\title{
Editorial:
}

\section{Important developments for the digital library: Data Ocean and Smart Library}

Yun-he PAN ${ }^{1,2}$, Editor-in-Chief of JZUS-C

( ${ }^{1}$ Zhejiang University, Hangzhou 310027, China)

$\left({ }^{2}\right.$ Chinese Academy of Engineering, Beijing 100088, China)

doi: $10.1631 /$ jzus.C1001000

Since its inception at Zhejiang University in 2005, the International Conference on the Universal Digital Library (ICUDL) has been held around the globe in Alexandria, Egypt, Carnegie Mellon University, USA, and at Allahabad in India. This annual event has been a strong driving force for exchange in digital library technologies, international scientific and cultural cooperation, as well as an influence on the development of the Universal Digital Library.

Now the sixth ICUDL is being held in Hangzhou (China) again, which is bound to become a good opportunity for us to summarize the past while casting our eyes into the future. The theme of this year's conference is 'Data Ocean and Cloud Computing'.

Nowadays, two major changes are taking place in digital library technologies. One is the transition from database into Data Ocean. This not only means a rapid increase in the number of books being digitalized, from 1 million to 5 million or even 10 million books, but also an enrichment in the forms of information, from books, pictures, calligraphy, videos, paintings and photos to a wide variety of relationships between them and their derivatives. The other change is the transition from digital library to Smart Library, where advanced services beyond the means of con-

(C) Zhejiang University and Springer-Verlag Berlin Heidelberg 2010 ventional libraries such as personalized services, hypertext services, computer-aided design (CAD) services, translation services, knowledge mining services, cross-media services are provided. This means that from the Data Ocean, new and various smart cloud services will arise to make the digital library more active, professional and intelligent.

It has been nearly ten years since the launching of the China-America Digital Academic Library (CADAL) with the ultimate goal of the digitalization all the library resources of humanity to allow everybody to access the knowledge, anywhere at any time. On completion of Phase I of CADAL, China has scanned over 1 million books in Chinese and English, which were released on the CADAL portal website for users from over 70 countries and regions, which is a great contribution to the conservation and sharing of human intellectual wealth. CADAL has already been marked as a milestone in the history of the Universal Digital Library.

It is my great pleasure to inform you that the Chinese Government has decided to invest 150 million Yuan to initiate Phase II of CADAL starting from April, 2010. The project will further increase the coverage and quantity of the

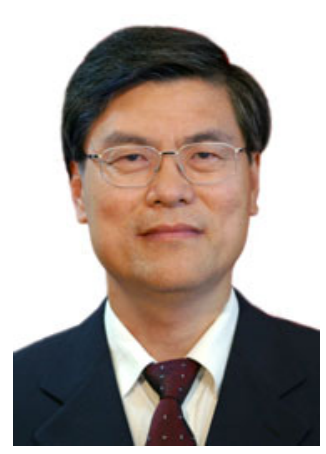

Prof. Yun-he PAN Editor-in-Chief Vice President, Chinese Academy of Engineering, China 
digital resources and provide for the digitalization of another 1.5 million books. As of today, over 60 Chinese higher education institutions have signed up for the digital resource construction. The project will further improve service capacities to provide data security and global services. We shall strengthen the technical support for the digital library and render the massive data contained in it into easy-to-use knowledge with technologies such as data mining, semantic analysis and cross-media techniques, build a large knowledge network, offer effective support for scientific research and improve education and learning. The project will involve novel system architecture and storage technology, knowledge mining technology, cross-media search and reasoning technology, active information service technology, automatic translation technology and new display technology for the digital library. Eventually, the project will further increase international cooperation and provide joint resource development and sharing, technical cooperation, personnel exchange, service customi- zation and sharing and other joint efforts on the basis of equality and mutual benefit.

Not long ago, the Digital Library Engineering Research Center of the Ministry of Education of China was established at Zhejiang University (Hangzhou, China). Faced with the exponential growth in digital resources and a massive increase in the number of users, higher demands have been placed upon digital library technologies and services, posing new challenges to its system efficiency, system security, information protection, and copyright protection. Through the research and development efforts, the center will become the core technology engine for the digital library in China, as well as a base for bringing together and raising talents in the field.

I believe, given the joint efforts of library and research institutions across the world and the potent support of computer technologies, the Smart Library will certainly become a reality. Let us work together for this dream of humanity! 